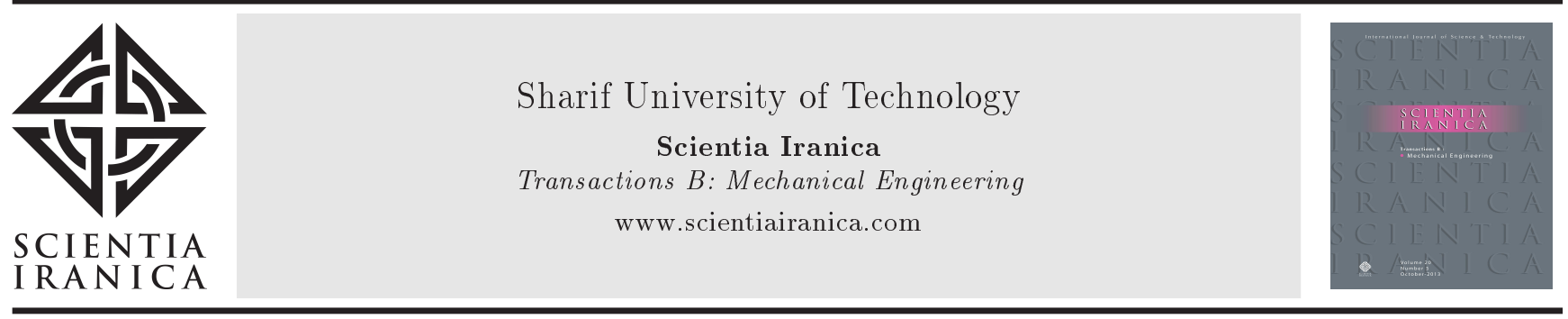

\title{
The importance of fluid-structure interaction simulation for determining the mechanical stimuli of endothelial cells and atheroprone regions in a coronary bifurcation
}

\author{
H.A. Pakravan, M.S. Saidi* and B. Firoozabadi \\ School of Mechanical Engineering, Sharif University of Technology, Tehran, Iran.
}

Received 8 June 2014; received in revised form 13 January 2015; accepted 14 April 2015

\section{KEYWORDS \\ Fluid-structure interaction; \\ Hemodynamics; \\ Coronary artery; \\ Wall shear stress; \\ Cyclic stretch; \\ Endothelial cells.}

\begin{abstract}
The function and morphology of Endothelial Cells (ECs) play a key role in atherosclerosis. The mechanical stimuli of ECs, such as Wall Shear Stress (WSS) and arterial wall strain, greatly influence the function and morphology of these cells. The present article deals with computations of these stimuli for a $3 \mathrm{D}$ model of a healthy coronary artery bifurcation. The focus of the study is to propose an accurate method for computations of WSS and strains. Two approaches are considered: Coupled simultaneous simulation of arterial wall and blood flow, called Fluid-Structure Interaction (FSI) simulation, and decoupled, which simulates each domain (fluid and solid domain) separately. The study demonstrates that the computed circumferential strains resulting from both methods are identical. However, longitudinal strain and WSS are very different from these two approaches. The resulting Time Averaged Wall Shear Stress (TAWSS) from the decoupled fluid model is always higher than the corresponding value from FSI simulation, while the Oscillatory Shear Index (OSI) from the rigid wall model is lower than the values resulting from FSI. Therefore, the decoupled simulation may underestimate the atheroprone sites of the artery, which suggests that using FSI simulation for mechanical stimuli of ECs is inevitable.
\end{abstract}

(C) 2016 Sharif University of Technology. All rights reserved.

\section{Introduction}

Atherosclerosis is a common disease and a major cause of morbidity and mortality worldwide [1]. Atherosclerosis does not occur randomly in arteries. Some arteries and locations are more prone to the disease [2], and coronary artery bifurcations are the most common places for this disease [2]. There is a close relationship between atherosclerosis and the geometry of the artery [3], the mechanical properties of the arterial

*. Corresponding author. Tel.: +98216616 5558; Fax: +982166000021

E-mail addresses: pakravan@mech.sharif.edu (H.A.

Pakravan); mssaidi@sharif.edu (M.S. Saidi);

firoozabadi@sharif.edu (B.Firoozabadi) wall [4], the hemodynamics of blood flow [5] and the shape and morphology of Endothelial Cells (ECs) [6]. ECs, which cover the luminal surface of the arterial wall, play an important role in atherosclerosis. The shape and morphology of these cells can suggest the atheroprone sites of the artery [2]. In the atheroprotected sites, ECs align and orient to the blood flow direction, while, in the atheroprone regions, they show no preferential orientation [7].

There is a strong relationship between the hemodynamics of blood flow and the morphology of ECs $[1,8,9]$. The cells orient and align to the direction of the one-way Wall Shear Stress (WSS) [7]. This orientation does not occur in low WSS regions [10]. On the other hand, ECs orient perpendicular to the direction of cyclic stretches of the arterial wall [11-13]. 
Due to the temporal changes of blood pressure, the arterial wall cyclically stretches in the circumferential direction and induces the ECs to orient parallel to the artery and flow direction. Therefore, these mechanical stimuli (WSS and cyclic strains of the arterial wall) could determine the shape and morphology of ECs and suggest atheroprone regions.

There are some techniques for in vivo measurement of arterial wall strains [14-16], as well as WSS $[17,18]$. Due to cardiac motion, these techniques are not accurate enough for coronary arteries [19]. In addition, the available approaches for in vivo measurement of WSS do not have enough accuracy for small arteries, such as coronary arteries $[17,18,20]$.

Due to lack of accurate methods for measuring WSS and strains, researchers use computational methods for determining these mechanical stimuli. Computational mechanical simulation of coronary arteries under realistic conditions involves some major complexities, which make it a difficult task. The coronary arteries are curved in their structure and have bifurcations. The arterial walls are flexible, and it seems that the rigid wall simulation is far from physiological conditions. The most important complexity in the simulation of coronary arteries is their dynamic curvature induced by cardiac motion during each heartbeat. This dynamic curvature could influence the mechanical stimuli of ECs. Some attempts were made to consider these complexities. Some studies $[8,21,22]$ simulated a coronary artery considering dynamic curvature and assuming a rigid wall to compute the WSS. In other studies $[1,23]$, the compliancy of the arterial wall was considered, and Fluid-Structure Interaction (FSI) simulation was employed. However, the dynamic curvature of the artery was neglected. There is a lack of studies regarding coronary artery simulations, including all its features, in the literature. Besides, studies dealing with rigid wall simulation $[8,21,22]$ do not explain the reasons for this assumption (i.e. rigid wall assumption). On the other hand, FSI simulation studies [1,23] do not completely describe the difference between the results obtained from FSI simulations and those from rigid wall simulations.

Based on the above review, in the present study, an attempt is made to model a Left Main coronary Artery (LMA) and its branches to the Left Anterior Descending (LAD) and left circumflex (LCX) arteries under physiological conditions, and considering dynamic curvature due to cardiac motion, to study the mechanical stimuli of ECs on different regions of the artery. The study focuses on the differences between FSI simulation and decoupled simulation of domains (i.e. arterial wall and blood flow, separately), and their effect on the mechanical stimuli of ECs. The present study attempts to find out whether FSI simulation is necessary for determining the mechanical stimuli of
ECs. It is obvious that if the decoupled simulation of domains has enough accuracy, simulation would be far easier and computationally more cost-effective.

\section{Methods}

The 3D geometry of LMA and its branches leading to LAD and LCX is reconstructed based on experimental measurements. Regarding measurements [24], the luminal diameters of these three branches is chosen as $3.8 \mathrm{~mm}, 3.1 \mathrm{~mm}$ and $2.9 \mathrm{~mm}$, respectively. A healthy coronary arterial wall, approximately, has $1 \mathrm{~mm}$ thickness [25], and this thickness is included in the geometry as the arterial wall. According to previous studies $[8,21,22]$, these branches are lying on a $25 \mathrm{~mm}$ radius sphere [26]. Usually, some straight segments are added to the ends of the model to mitigate the uncertainty in boundary conditions [27-29]. Based on the study of Liu et al. [30], a $27 \mathrm{~mm}$ straight segment is added to LMA and two $15 \mathrm{~mm}$ ones are added to the two other ends. The reconstructed geometry is shown in Figure 1. Similar to previous studies $[8,21,29]$, the effect of dynamic curvature due to cardiac motion is applied using a sinusoidal time varying function. The applied displacement, $d$, of three ends normal to the artery axis is as follows:

$$
\frac{d}{R_{0}}=-\delta \sin \frac{2 \pi t}{T}
$$

where $R_{0}$ is the initial radius of the sphere and equal to $25 \mathrm{~mm} ; \delta$ is a parameter related to the intensity of cardiac motion and dynamic curvature; $t$ is time; and $T$ is the period of a heart cycle. For a $60 \mathrm{bpm}$ heart rate, which is used in this study, it is equal to $1 \mathrm{~s}$.

Usually, for FSI simulation, a combination of pressure-velocity boundary conditions is required [28]. Physiological measured velocity [31] is used as the inlet, boundary condition at the LMA inlet and physiological measured pressures [31] are used as outlet boundary conditions at LAD and LCX outlets. For the solid domain, the specified displacement of Eq. (1) is applied to the three ends of the solid model.

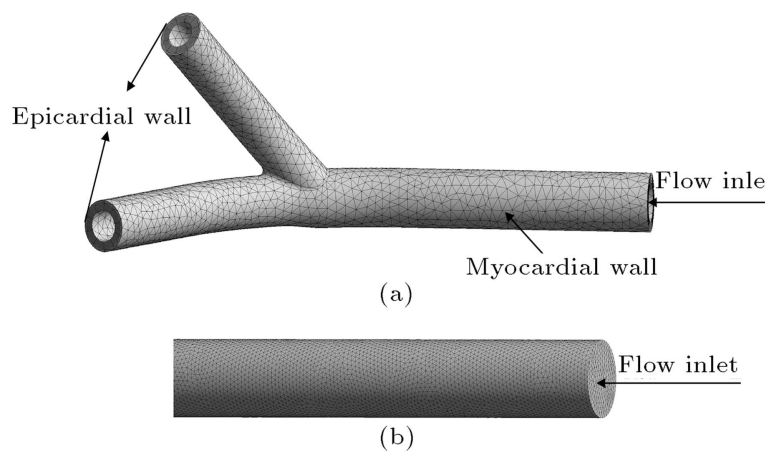

Figure 1. The geometry of the artery and the generated meshes for (a) solid domain, and (b) fluid domain at LMA inlet. 
It is reported $[32,33]$ that the blood in vessels with diameters larger than $1 \mathrm{~mm}$ shows Newtonian behavior. In the current simulation, blood is modeled as an incompressible, Newtonian fluid, with $1050 \mathrm{~kg} / \mathrm{m}^{3}$ for density $[1,27,28]$ and 0.004 Pa.s for viscosity $[1,8,9]$. The arterial wall is a multilayer, anisotropic structure, but, in many simulations $[23,28,34,35]$, it is considered an isotropic single-layer solid. Also, some studies $[29,36,37]$ describe the mechanical properties of the arterial wall using the Mooney-Rivlin model. In the present study, the arterial wall is considered an isotropic single-layer solid using the Mooney-Rivlin model. Constants of the Mooney-Rivlin model are determined based on a stress-strain curve resulting from experimental measurement [29] of a coronary artery in the circumferential direction. The achieved strain energy density function is as follows:

$$
\begin{aligned}
W_{D}= & -1.47 \times 10^{3}\left(I_{1}-3\right)+1.5478 \times 10^{3}\left(I_{2}-3\right) \\
& +6.4071 \times 10^{4}\left(I_{1}-3\right)^{2}-1.5178 \\
& \times 10^{5}\left(I_{1}-3\right)\left(I_{2}-3\right)+9.1591 \\
& \times 10^{4}\left(I_{2}-3\right)^{2}+10^{7}(J-1)^{2},
\end{aligned}
$$

where $I_{1}$ and $I_{2}$ are invariants and $J$ is the determinant of the Cauchy-Green deformation tensor. Also, the considered density for the arterial wall is 1060 $\mathrm{kg} / \mathrm{m}^{3}[23]$.

Different meshes consisting of 135000, 288000, 525000 , and 968000 cells were considered for the fluid domain. For each mesh size, WSS on the myocardial and epicardial line, as well as the velocity distribution on the LAD outlet, were investigated. The grid independent study showed that the results for 525000 cells and 968000 cells were identical. Therefore, all simulations are performed using a mesh size of 525000 cells, which is adequate for this study. Different mesh sizes, with 1 to 8-layers of cells along the thickness of the arterial wall, were studied for the solid domain as well. The results showed that the computed circumferential and longitudinal strains using a mesh size with 7 layers of cells are equal to those achieved from an 8-layer mesh. So, a mesh size with 7 layers of cells along the thickness which has 23000 cells is used for all simulations. Figure 1 shows the reconstructed geometry of the present study with the generated meshes for each domain.

To study the importance of FSI simulation for computation of the mechanical stimuli of ECs, simulations are performed based on the two approaches. In the first approach, the fluid and solid domains are coupled and FSI simulation is used to simultaneously compute the experienced WSS and strains by ECs. It should be noticed that both the arterial wall (solid domain) and blood flow (fluid domain) must be solved to determine the mechanical stimuli of ECs. The second approach assumes that solid and fluid domains are decoupled and each phase is separately solved. In this approach, the fluid domain is independently solved, while the arterial wall is assumed rigid. At this stage, only WSS can be computed. To compute cyclic stretches, the solid domain is separately solved. It is obvious that both approaches can compute the mechanical stimuli of ECs. However, the decoupled simulation needs less computational effort. On the other hand, the FSI simulation has more computational cost, as well as more difficulties, which may provide more accurate results.

The governing equations for the fluid domain are Navier-Stokes and continuity equations and for the solid domain, the equilibrium equation is used. ANSYS-Structural (FEM software for the solid domain) and ANSYS-Fluent (FVM software for the fluid domain), available in ANSYS Workbench v14.5, were used for all simulations. Fluent employs an Arbitrary Lagrangian-Eulerian (ALE) formulation for moving wall problems. The SIMPLE algorithm was used for pressure-velocity coupling and the second order upwind scheme for discretization of convective terms of the momentum equations. The solid domain was simulated as a large deformation problem and a nonlinear stabilization procedure was employed for better convergence. The time step for all simulations is 0.01 sec. A personal computer, with Core i7-3770 CPU (3.40 GHz) and 16.0 GB of RAM, was used for all simulations. The simulations were performed in a parallel manner with 7 core of CPU. The computational time is, on average, 31 hours for FSI simulations and 13 hours for decoupled simulations (about 9 hours for the fluid domain plus 4 hours for the solid domain).

\section{Results and discussion}

\subsection{Validation of the results}

Simulations were performed using the methods described in Section 2. To validate the results of the simulations, some quantities are compared with available results in literature. Figure 2 compares the results of the present FSI simulation with previous ones. Figure 2(a) illustrates the computed blood pressure at the LMA inlet from the present study, along with the experimental measurements of Davies et al. [31], which shows good agreement between our predicted results and experimental measurements. Figure 2(b) demonstrates the temporal variation of maximum WSS in the artery from our simulation results and is compared with simulation results obtained by Malvè et al. [28]. These two results show the same trends and close values. Boundary conditions in our simulation and that of the Malvè et al. [28] simulation are the same. However, the 


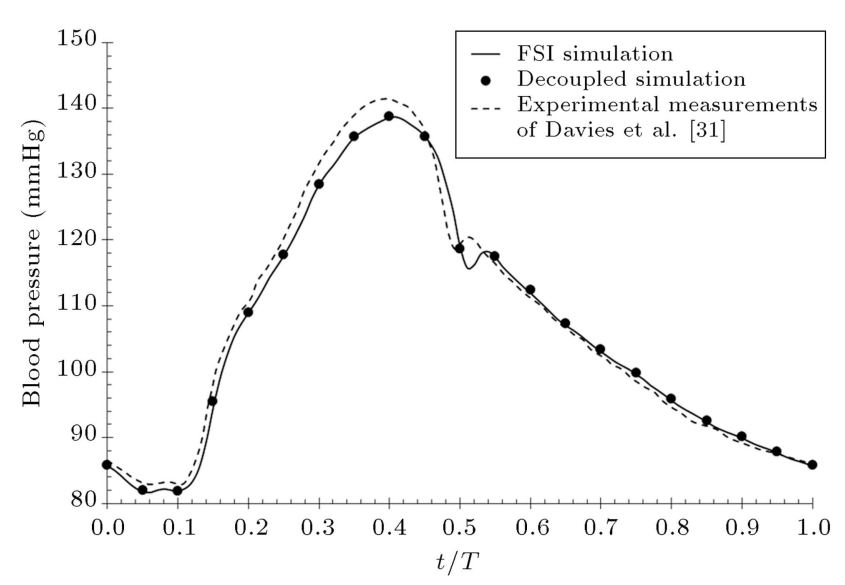

(a)

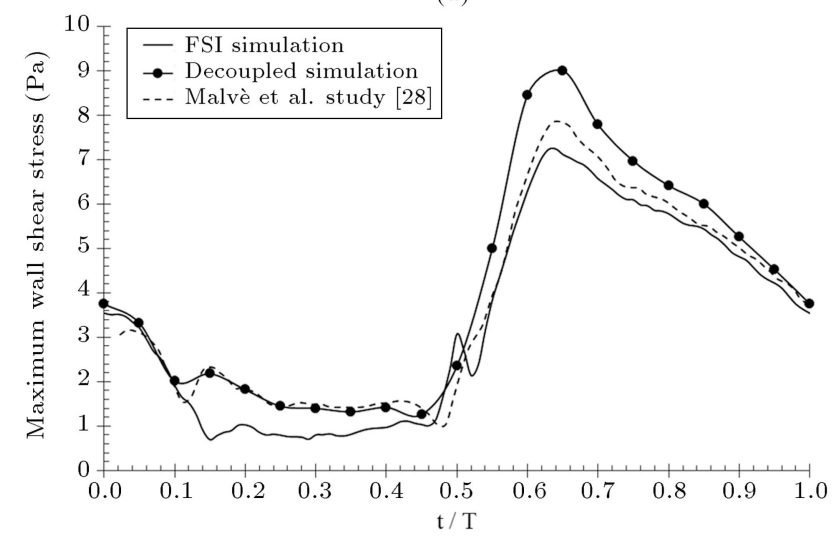

(b)

Figure 2. Validation of the results: (a) Comparison of computed blood pressure (in $\mathrm{mmHg}$ ) at LMA inlet with experimental measurements of Davies et al. [31]; and (b) comparison of computed maximum average circumferential stress (in $\mathrm{Pa}$ ) of LMA with results obtained by Malvè et al. [28].

geometry and mechanical properties of the arterial wall are different, which creates a responsibility for some discrepancies between results. The maximum average circumferential strain is another important mechanical stimulus, which is computed in the current study. The computed value in the present simulation (for both FSI and decoupled simulations) is $5.4 \%$ for LMA, which is in agreement with $4 \pm 1.8 \%$ measured by Ding and Friedman [26] and 5\% reported by Qiu and Tarbell [38]. Following these validations, the simulations could be performed and the results could be investigated. The mechanical stimuli of ECs are WSS and cyclic strains in longitudinal and circumferential directions, which are separately presented in this section. For accurate investigation of the results, 21 locations on the arterial wall are considered and mechanical stimuli of ECs in these regions are studied. These locations are chosen in such a way that different segments and behaviors of the mechanical stimuli of ECs would be covered. The locations are shown in Figure 3 on the coronary artery model.

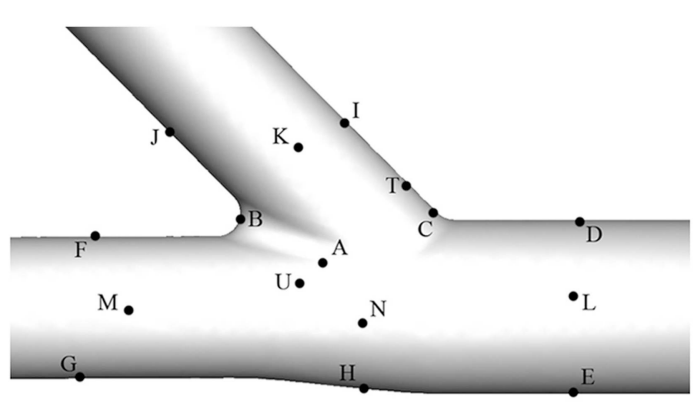

(a)

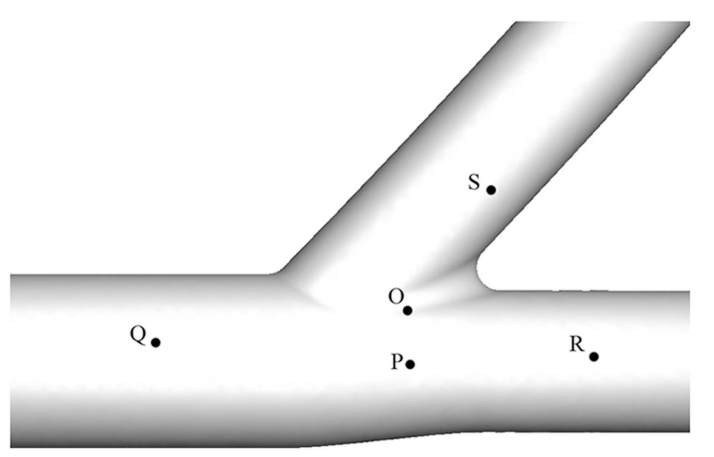

(b)

Figure 3. Different locations for investigation on (a) myocardial wall, and (b) epicardial wall of the artery.

\subsection{Cyclic stretches}

Cyclic stretches of the arterial wall which are exerted on ECs may influence the cell functions. These stretches in two directions are important: circumferential and longitudinal. Circumferential strains induce the ECs to orient and align parallel to the blood flow, while longitudinal strains induce the cells to orient and align perpendicular to the blood flow [11-13]. Therefore, these mechanical stimuli are a concern of interest. As stated above, strains could be computed using FSI simulation or decoupled simulation for the solid domain. For the latter approach, blood pressure can be used as the boundary condition on the luminal surface of the arterial wall. In FSI simulation, the data that is transferred from the fluid to the solid domain is the stress vector on the wall in each node. Using hydrostatic blood pressure as the boundary condition on the luminal surface of the artery instead of the stress tensor may introduce some errors. However, since shear stresses are some orders of magnitude smaller than hydrostatic blood pressure, this approximation seems reasonable. In the next step, the temporal and spatial variations of blood pressure as the boundary condition should be determined. Figure 4 shows the pressure distribution on the luminal surface of the arterial surface, which is computed from FSI simulation at two instances of heart cycle corresponding to minimum blood pressure (Figure 4(a)) and maximum blood pressure (Figure 4(b)). Figure 4 demonstrates that the spatial variation of blood pressure at each time is 

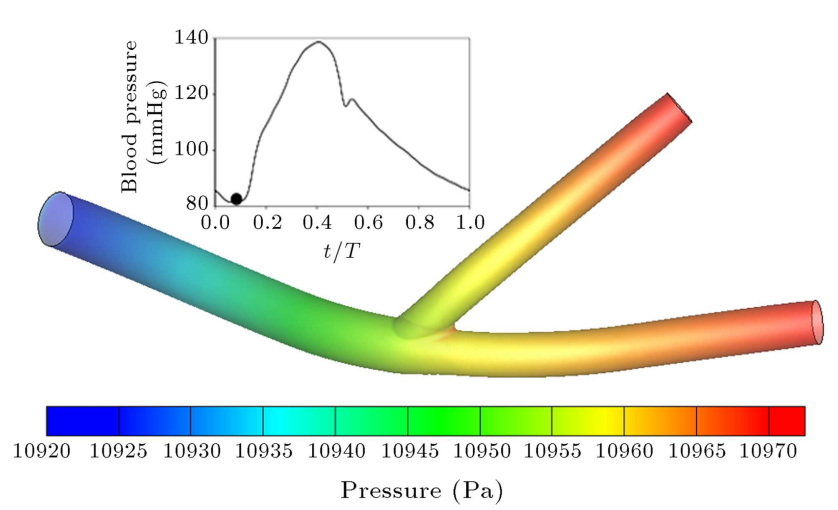

(a)
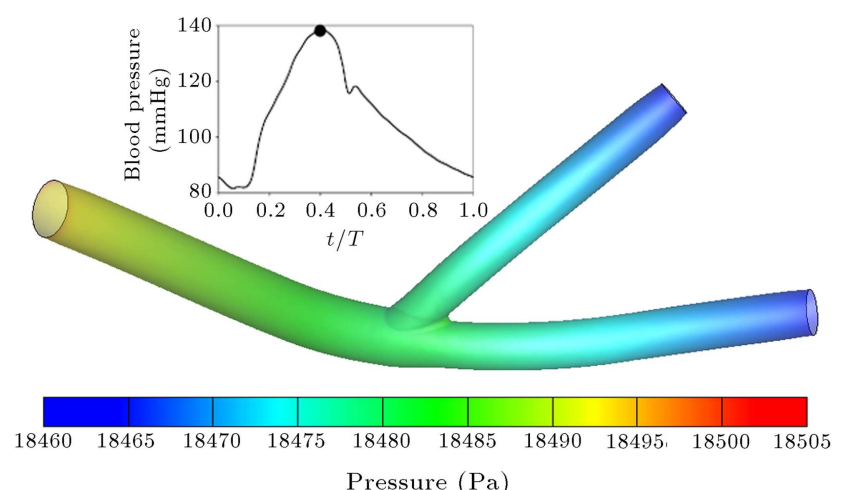

(b)

Figure 4. Hydrostatic blood pressure distribution (in $\mathrm{Pa}$ ) at instants (a) $t=0.1 T$, and (b) $t=0.4 T$.

less than $0.5 \%$. Therefore, the blood pressure at the luminal surface of the artery for simulation of the solid domain could be accurately approximated as uniform pressure along the entire artery, and only the temporal variation of blood pressure should be considered. So, simulation of the solid domain is performed using blood pressure as the boundary condition on the luminal surface of the artery to compare the resulted strains with those from the FSI simulation.

The maximum circumferential and longitudinal strains of endothelium in a heart cycle are presented in Table 1. The values are computed for cases with (corresponding to $\delta=0.2$ in Eq. (1)), and without dynamic curvature due to cardiac motion. Besides, for each case, the simulation are done for two approaches: FSI simulation and decoupled simulation of the solid domain. The results of Table 1 show that the maximum circumferential strain computed from the FSI simulation are nearly equal to those computed from the solid simulation. The difference between these two values is up to $4 \%$. However, this difference for longitudinal strain could be too large. The maximum error in the decoupled solid simulation for computation of longitudinal strain compared to FSI simulation could be as large as $134 \%$ in cases without dynamic curvature and $98 \%$ in cases with dynamic curvature. The cause of this difference is that the effect of WSS resulted from blood flow is important on the longitudinal strain. These WSSs are neglected in the decoupled solid simulation and the blood pressure boundary condition of this simulation cannot apply this effect to the solid domain. On the other hand, the effect of hydrostatic blood pressure on the circumferential strain of the endothelium is far more than the effect of WSS. Therefore, the decoupled solid simulation from the fluid domain can accurately compute the circumferential strain of the arterial wall and endothelium, and is more computationally costeffective than FSI simulation. Nevertheless, for longitudinal strain, the error in the decoupled solid simulation is too large, so, using the FSI simulation is necessary in this case.

Table 1 also shows that considering dynamic curvature can increase the longitudinal strain of ECs, which induces their inappropriate orientation. Locations such as K, L and M, which have high longitudinal strain, might be prone to atherosclerosis.

\subsection{Wall shear stresses}

The WSS, as an important mechanical stimulus of ECs, is presented in this sub-section. This parameter is computed from two approaches: FSI simulation and decoupled fluid domain simulation. The results of these approaches are compared to investigate the effects of FSI simulation. Figure 5 shows the effect of wall flexibility on the WSS variation of some indicated locations on Figure 3. It can be seen that for all shown locations in Figure 5, the minimum WSS in the heart cycle that resulted from FSI simulation (compliant wall mode) is less than the corresponding value in the rigid wall simulation. The effects of the compliant wall model on the maximum WSS in the heart cycle are not identical for all locations. For locations D, G and N (Figure 5(a), (b) and (d)), the maximum WSS that resulted from rigid wall simulation is larger than the corresponding value of FSI simulation. However, this trend for locations I and $\mathrm{T}$ (Figure 5(c) and (e)) is reverse, i.e. the maximum WSS for these two locations that resulted from rigid wall simulation is larger than FSI simulation, and, finally, this value for location U (Figure $5(\mathrm{f})$ ) is the same for the two models. In addition, Figure 5 shows that the effect of wall compliancy is stronger than the dynamic curvature of the artery due to cardiac motion.

For a more accurate and quantitative investigation of the mechanical stimuli of ECs and their effects on functions of these cells, some other parameters may be introduced. Time Averaged Wall Shear Stress (TAWSS) and the Oscillatory Shear Index (OSI) are important parameters related to WSS for studying the mechanical stimuli of ECs and atherosclerosis, which are defined in Eqs. (3) and (4) [1,39]: 
Table 1. Comparison of longitudinal and circumferential strains (in \%) for different simulation methods.

\begin{tabular}{|c|c|c|c|c|c|c|c|c|}
\hline \multirow{3}{*}{ Location } & \multicolumn{4}{|c|}{ Without dynamic curvature } & \multicolumn{4}{|c|}{ With dynamic curvature } \\
\hline & \multicolumn{2}{|c|}{$\mathrm{FSI}^{+}$} & \multicolumn{2}{|c|}{ Solid $^{++}$} & \multicolumn{2}{|c|}{ FSI } & \multicolumn{2}{|c|}{ Solid } \\
\hline & Circ.* & Long.** & Circ. & Long. & Circ. & Long. & Circ. & Long. \\
\hline A & 2.33 & 1.24 & 2.35 & 2.23 & 5.62 & 3.27 & 5.50 & 3.61 \\
\hline $\mathrm{B}$ & 4.89 & 1.75 & 4.81 & 1.73 & 4.88 & 1.77 & 4.76 & 1.73 \\
\hline $\mathrm{C}$ & 5.42 & 1.99 & 5.28 & 1.90 & 5.44 & 2.17 & 5.24 & 2.08 \\
\hline $\mathrm{D}$ & 5.52 & 0.11 & 5.56 & 0.16 & 5.62 & 4.87 & 5.66 & 5.05 \\
\hline $\mathrm{E}$ & 5.30 & 0.63 & 5.34 & 0.62 & 5.39 & 1.84 & 5.42 & 1.70 \\
\hline $\mathrm{F}$ & 5.68 & 0.03 & 5.73 & 0.02 & 5.82 & 2.92 & 5.85 & 3.01 \\
\hline G & 5.38 & 0.74 & 5.44 & 0.71 & 5.38 & 1.52 & 5.42 & 1.35 \\
\hline $\mathrm{H}$ & 5.29 & 0.60 & 5.31 & 0.49 & 5.40 & 1.74 & 5.38 & 1.51 \\
\hline I & 5.14 & 0.19 & 5.18 & 0.06 & 5.23 & 3.77 & 5.25 & 3.92 \\
\hline $\mathrm{J}$ & 5.32 & 0.33 & 5.33 & 0.28 & 5.46 & 0.64 & 5.44 & 0.68 \\
\hline $\mathrm{K}$ & 5.42 & 0.21 & 5.58 & 0.02 & 5.52 & 6.80 & 5.70 & 6.73 \\
\hline $\mathrm{L}$ & 5.79 & 0.30 & 5.78 & 0.00 & 6.81 & 9.82 & 6.76 & 0.10 \\
\hline M & 5.77 & 0.07 & 5.85 & 0.16 & 5.95 & 6.79 & 6.02 & 6.85 \\
\hline $\mathrm{N}$ & 5.18 & 0.57 & 5.16 & 0.41 & 5.56 & 8.04 & 5.50 & 8.12 \\
\hline $\mathrm{O}$ & 3.32 & 0.70 & 3.37 & 1.25 & 4.20 & 0.85 & 4.32 & 1.42 \\
\hline $\mathrm{P}$ & 4.84 & 1.48 & 4.74 & 1.46 & 4.57 & 4.19 & 4.47 & 4.47 \\
\hline $\mathrm{Q}$ & 5.35 & 1.07 & 5.35 & 1.08 & 5.33 & 4.40 & 5.33 & 4.47 \\
\hline $\mathrm{R}$ & 5.33 & 0.99 & 5.38 & 1.10 & 5.08 & 3.61 & 5.11 & 4.00 \\
\hline $\mathrm{S}$ & 5.42 & 0.71 & 5.52 & 0.80 & 5.33 & 2.77 & 5.45 & 2.79 \\
\hline $\mathrm{T}$ & 5.17 & 0.61 & 5.12 & 0.74 & 5.21 & 3.13 & 5.13 & 3.28 \\
\hline $\mathrm{U}$ & 5.63 & 0.35 & 5.83 & 0.44 & 5.82 & 4.66 & 6.04 & 4.77 \\
\hline
\end{tabular}

$$
\begin{aligned}
& \text { TAWSS }=\frac{1}{T} \int_{0}^{T}|\boldsymbol{\tau}| d t, \\
& \text { OSI }=0.5\left(1-\frac{\left|\int_{0}^{T} \tau d t\right|}{\int_{0}^{T}|\tau| d t}\right),
\end{aligned}
$$

where $\boldsymbol{\tau}$ is the WSS vector and $T$ is the period of a heart cycle. Regions with low TAWSS and high OSI are known as atheroprone sites. TAWSS smaller than $0.5 \mathrm{~Pa}$ and OSI larger than 0.2 could be an approximate border for atherosclerosis [40]. Table 2 shows, in detail, these parameters for different cases. These cases include considering the dynamic curvature of the artery due to cardiac motion $(\delta=0.2$ in Eq. (1)) and neglecting this effect. The simulations for each are performed using FSI simulation and the decoupled fluid model. The results of Table 2 demonstrate that neglecting wall compliancy (decoupled fluid simulation) in both conditions (i.e. with and without dynamic curvature) may overestimate the TAWSS on ECs up to $88 \%$, compared to FSI simulation. However, TAWSS for locations lying on straight segments like R, F, $\mathrm{J}$, and $\mathrm{K}$ are nearly equal for FSI and rigid wall simulations (with up to $15 \%$ difference). On the other hand, the predicted values of OSI, in the case of neglecting the dynamic curvature of the artery, from decoupled fluid simulation, are up to $91 \%$ lower than those resulted from FSI simulation. This error, in cases of considering dynamic curvature, is $78 \%$. Therefore, since the TAWSS in the decoupled fluid simulation is higher than FSI, and OSI in the decoupled fluid simulation is lower than FSI, the rigid wall assumption may suggest that some regions, which are prone to the disease, are atheroprotected. In addition, Table 2 shows that the most critical locations are $\mathrm{T}$ and $\mathrm{U}$, which are placed on the myocardial side of the bifurcation. Location $\mathrm{T}$ has lower TAWSS and higher OSI, while, for location U, TAWSS is higher, OSI is lower, and both values for both locations are in the critical range. 


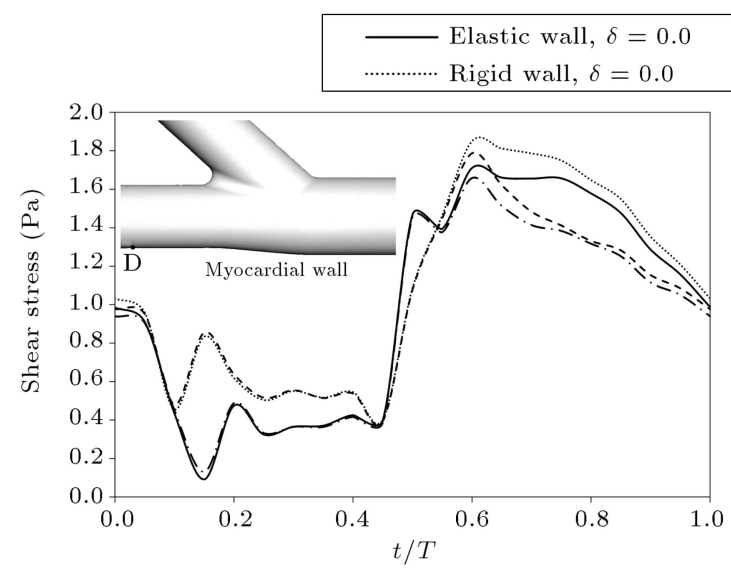

(a)

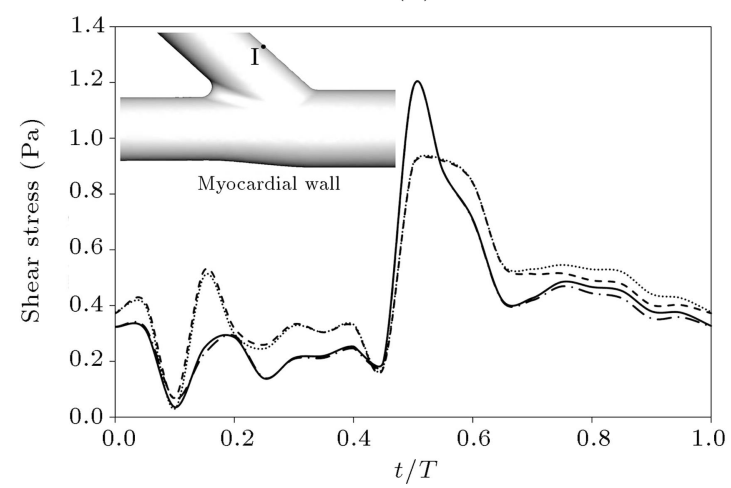

(c)

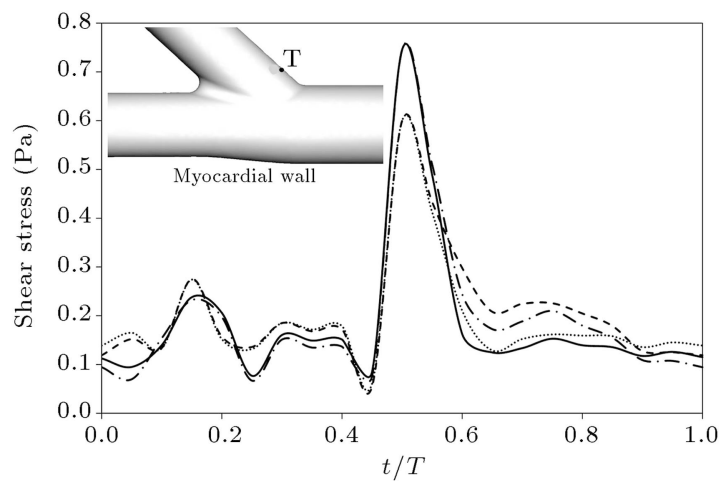

(e)

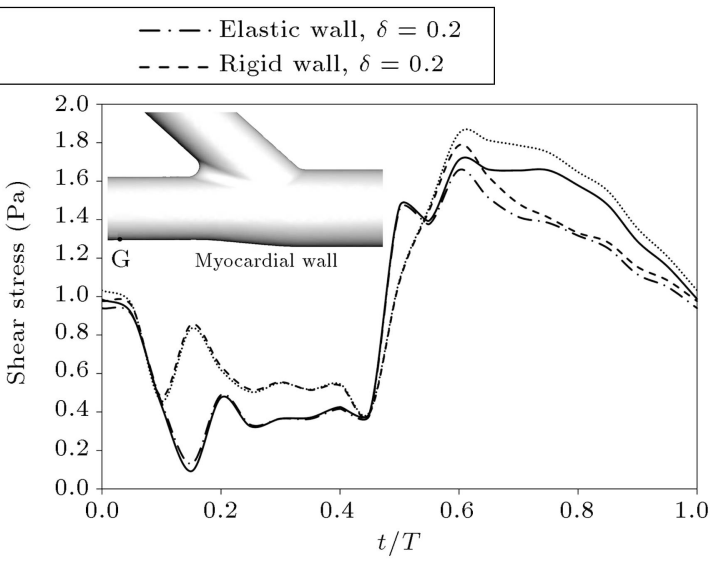

(b)

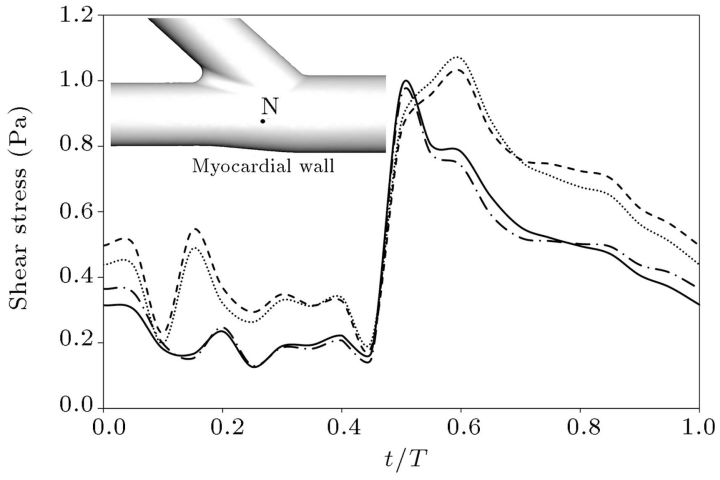

(d)

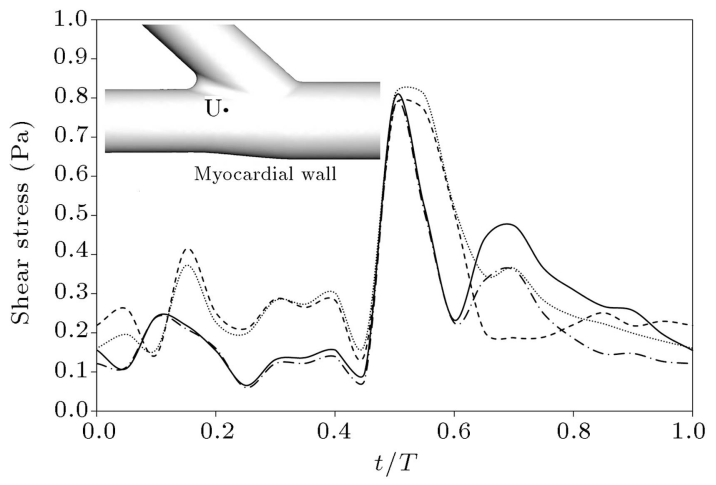

(f)

Figure 5. Effect of elastic or rigid wall simulation on WSS variations (in Pa) in a heart cycle for (a) location D, (b) location G, (c) location I, (d) location N, (e) location T, and (f) location U.

\section{Conclusion}

In the present study, the bifurcation of LMA to LAD and LCX was simulated using FSI and decoupled simulation of each domain to determine the mechanical stimuli of ECs (wall shear stress and strains in circumferential and longitudinal directions). The idealized 3D model of the artery was reconstructed based on the experimental measurements of the coronary arteries. This geometry is a simplification of the real geometry. Although the model reconstructed based on CT images is usually more accurate than those of the ideal, special attention should be paid in cases of FSI simulations.
The CT images are captured in a pressurized state (when blood pressure exists inside the artery); however, the structural analysis for solids must be initiated from the zero-stress state (the configuration without any structural stresses). Therefore, using CT images for FSI simulations could create large errors in results. Recently, Vavourakis et al. [41] presented an approach for reconstructing a geometry in the zero-stress state from pressurized CT images. However, still, many studies mistakenly used the models reconstructed from CT images in their FSI simulations without any corrections. The physiological pressure and flow velocities were used as the boundary conditions. In addition, 
Table 2. Comparison TAWSS (in Pa) and OSI (dimensionless) for different simulation methods.

\begin{tabular}{|c|c|c|c|c|c|c|c|c|}
\hline \multirow{3}{*}{ Location } & \multicolumn{4}{|c|}{ Without dynamic curvature } & \multicolumn{4}{|c|}{ With dynamic curvature } \\
\hline & \multicolumn{2}{|c|}{ Compliant } & \multicolumn{2}{|c|}{ Rigid } & \multicolumn{2}{|c|}{ Compliant } & \multicolumn{2}{|c|}{ Rigid } \\
\hline & TAWSS & OSI & TAWSS & OSI & TAWSS & OSI & TAWSS & OSI \\
\hline A & 0.795 & 0.029 & 1.208 & 0.023 & 0.779 & 0.026 & 1.188 & 0.017 \\
\hline $\mathrm{B}$ & 3.358 & 0.005 & 4.070 & 0.002 & 3.496 & 0.004 & 4.316 & 0.003 \\
\hline $\mathrm{C}$ & 0.850 & 0.009 & 1.052 & 0.001 & 0.849 & 0.010 & 1.075 & 0.005 \\
\hline $\mathrm{D}$ & 1.003 & 0.002 & 1.184 & 0.001 & 0.979 & 0.001 & 1.156 & 0.001 \\
\hline $\mathrm{E}$ & 0.859 & 0.003 & 1.054 & 0.002 & 0.821 & 0.002 & 1.013 & 0.001 \\
\hline $\mathrm{F}$ & 1.212 & 0.001 & 1.396 & 0.001 & 1.283 & 0.003 & 1.483 & 0.003 \\
\hline G & 0.991 & 0.002 & 1.093 & 0.001 & 0.917 & 0.004 & 1.004 & 0.004 \\
\hline $\mathrm{H}$ & 0.678 & 0.014 & 0.825 & 0.008 & 0.588 & 0.011 & 0.724 & 0.005 \\
\hline I & 0.397 & 0.035 & 0.457 & 0.003 & 0.391 & 0.039 & 0.452 & 0.011 \\
\hline $\mathrm{J}$ & 1.228 & 0.002 & 1.356 & 0.001 & 1.225 & 0.004 & 1.345 & 0.004 \\
\hline $\mathrm{K}$ & 0.763 & 0.014 & 0.843 & 0.001 & 0.768 & 0.016 & 0.857 & 0.006 \\
\hline $\mathrm{L}$ & 0.782 & 0.001 & 0.975 & 0.000 & 0.807 & 0.001 & 1.018 & 0.001 \\
\hline $\mathrm{M}$ & 0.859 & 0.001 & 0.940 & 0.000 & 0.854 & 0.003 & 0.952 & 0.003 \\
\hline $\mathrm{N}$ & 0.402 & 0.053 & 0.545 & 0.025 & 0.404 & 0.043 & 0.567 & 0.016 \\
\hline $\mathrm{O}$ & 0.964 & 0.010 & 1.811 & 0.005 & 0.874 & 0.013 & 1.638 & 0.005 \\
\hline $\mathrm{P}$ & 0.680 & 0.005 & 1.128 & 0.002 & 0.526 & 0.005 & 0.947 & 0.002 \\
\hline $\mathrm{Q}$ & 0.933 & 0.001 & 1.132 & 0.000 & 0.898 & 0.001 & 1.109 & 0.001 \\
\hline $\mathrm{R}$ & 1.301 & 0.001 & 1.367 & 0.001 & 1.252 & 0.001 & 1.294 & 0.002 \\
\hline $\mathrm{S}$ & 1.650 & 0.002 & 1.697 & 0.001 & 1.568 & 0.004 & 1.601 & 0.005 \\
\hline $\mathrm{T}$ & 0.184 & 0.146 & 0.190 & 0.063 & 0.192 & 0.135 & 0.205 & 0.067 \\
\hline $\mathrm{U}$ & 0.261 & 0.294 & 0.309 & 0.149 & 0.217 & 0.295 & 0.298 & 0.066 \\
\hline
\end{tabular}

the dynamic curvature of the artery due to cardiac motion was applied to the model. The article focused on investigating the importance of FSI simulation for determining the mechanical stimuli of ECs. Two approaches were used for this study: FSI simulation which solves the solid (arterial wall) and fluid (blood flow) domains in a coupled manner, and decoupled simulation which solves each domain separately. The former may have higher accuracy and more computational cost, while the latter is simpler and computationally more cost-effective, but is less accurate. The WSS and circumferential and longitudinal strains were computed using both methods. The results indicated that the decoupled solid simulation using blood pressure as the inside boundary condition can accurately predict the circumferential strain as FSI simulation. However, this kind of simulation failed to accurately compute the longitudinal strains and could induce up to $134 \%$ error. On the other hand, the decoupled fluid domain simulation may make $90 \%$ error in predicting TAWSS and OSI. The computed value of TAWSS from fluid domain simulation, which is equivalent to rigid wall simulation, is higher than the compliant model (FSI simulation), while the values of OSI from decoupled fluid simulation is lower than the corresponding value achieved from FSI simulation. Therefore, the rigid wall model can miss some atheroprone sites. These results conclude that FSI simulation is inevitable for computation of the mechanical stimuli of ECs, and the decoupled simulations of each phase could greatly influence the results. However, for some problems that are commonly seen in the literature, such as wall shear stress computation in arteries without bifurcation, the rigid wall simulation is adequate. In addition, for circumferential strain computation in arterial walls, the decoupled solid simulation works adequately. For these problems, FSI simulation is not only unnecessary, but is simply a time- and cost-consuming task.

\section{References}

1. Torii, R., Wood, N.B., Hadjiloizou, N., Dowsey, A.W., Wright, A.R., Hughes, A.D., Davies, J., Francis, D.P., Mayet, J. and Yang, G.Z. "Fluid-structure interaction analysis of a patient-specific right coronary artery with physiological velocity and pressure waveforms", Communications in Numerical Methods in Engineering, 25(5), pp. 565-580 (2009).

2. Nerem, R.M., Levesque, M.J. and Cornhill, J. "Vascular endothelial morphology as an indicator of the 
pattern of blood flow", J. Biomech. Eng., 103(3), pp. 172 (1981).

3. Phan, T.G., Beare, R.J., Jolley, D., Das, G., Ren, M., Wong, K., Chong, W., Sinnott, M.D., Hilton, J.E. and Srikanth, V. "Carotid artery anatomy and geometry as risk factors for carotid atherosclerotic disease", Stroke, 43(6), pp. 1596-1601 (2012).

4. Li, M., Beech-Brandt, J., John, L., Hoskins, P. and Easson, W. "Numerical analysis of pulsatile blood flow and vessel wall mechanics in different degrees of stenoses", J. Biomech., 40(16), pp. 3715-3724 (2007).

5. Helmlinger, G., Geiger, R., Schreck, S. and Nerem, R. "Effects of pulsatile flow on cultured vascular endothelial cell morphology", J. Biomech. Eng., 113(2), pp. 123-131 (1991).

6. Kratky, R.G. and Roach, M.R. "Relationship between aortic endothelial cell morphology and atherosclerosis in rabbits", Scan. Electron Microsc. (Pt 3), p. 1461 (1983).

7. Ohashi, T. and Sato, M. "Remodeling of vascular endothelial cells exposed to fluid shear stress: experimental and numerical approach", Fluid Dynamics Research, 37(1), pp. 40-59 (2005).

8. Weydahl, E.S. and Moore, J.E. "Dynamic curvature strongly affects wall shear rates in a coronary artery bifurcation model", J. Biomech., 34(9), pp. 1189-1196 (2001).

9. Bazilevs, Y., Hsu, M.-C., Zhang, Y., Wang, W., Kvamsdal, T., Hentschel, S. and Isaksen, J. "Computational vascular fluid-structure interaction: Methodology and application to cerebral aneurysms", Biomechanics and Modeling in Mechanobiology, 9(4), pp. 481-498 (2010).

10. Franke, R.-P., Gräfe, M., Schnittler, H., Seiffge, D., Mittermayer, C. and Drenckhahn, D. "Induction of human vascular endothelial stress fibres by fluid shear stress", Nature, 307, pp. 648-649 (1984).

11. Kang, J., Steward, R.L., Kim, Y., Schwartz, R.S., Leduc, P.R. and Puskar, K.M. "Response of an actin filament network model under cyclic stretching through a coarse grained Monte Carlo approach", J. Theor. Biol., 274(1), pp. 109-119 (2011).

12. Stamenović, D., Lazopoulos, K.A., Pirentis, A. and Suki, B. "Mechanical stability determines stress fiber and focal adhesion orientation", Cellular and Molecular Bioengineering, 2(4), pp. 475-485 (2009).

13. Yamada, H., Takemasa, T. and Yamaguchi, T. "Theoretical study of intracellular stress fiber orientation under cyclic deformation", J. Biomech., 33(11), pp. 1501-1505 (2000).

14. Squire, M.J., Rogers, C. and Edelman, E.R. "Measuring arterial strain induced by endovascular stents", Med. Biol. Eng. Comput, 37(6), pp. 692-698 (1999).

15. Lin, A.P., Bennett, E., Wisk, L.E., Gharib, M., Fraser, S.E. and Wen, H. "Circumferential strain in the wall of the common carotid artery: Comparing displacementencoded and cine MRI in volunteers", Magn. Reson. Med., 60(1), pp. 8-13 (2008).
16. Larsson, M., Kremer, F., Claus, P., Kuznetsova, T., Brodin, L. and D'hooge, J. "Ultrasound-based radial and longitudinal strain estimation of the carotid artery: A feasibility study", Ultrasonics, Ferroelectrics and Frequency Control, IEEE Transactions on, 58(10), pp. 2244-2251 (2011).

17. Van der Giessen, A.G., Wentzel, J.J., Meijboom, W.B., Mollet, N.R., Van Der Steen, A.F., Van De Vosse, F.N., De Feyter, P.J. and Gijsen, F.J. "Plaque and shear stress distribution in human coronary bifurcations: A multislice computed tomography study", EuroIntervention, 4(5), pp. 654-661 (2009).

18. Shaaban, A.M. and Duerinckx, A.J. "Wall shear stress and early atherosclerosis: A review", Am. J. Roentgenol., 174(6), pp. 1657-1665 (2000).

19. Liang, Y., Zhu, H., Gehrig, T. and Friedman, M.H. "Measurement of the transverse strain tensor in the coronary arterial wall from clinical intravascular ultrasound images", J. Biomech., 41(14), pp. 2906-2911 (2008).

20. Van der Giessen, A.G., Groen, H.C., Doriot, P.-A., De Feyter, P.J., Van Der Steen, A.F., Van De Vosse, F.N., Wentzel, J.J. and Gijsen, F.J. "The influence of boundary conditions on wall shear stress distribution in patients specific coronary trees", J. Biomech., 44(6), pp. 1089-1095 (2011).

21. Pivkin, I., Richardson, P., Laidlaw, D. and Karniadakis, G. "Combined effects of pulsatile flow and dynamic curvature on wall shear stress in a coronary artery bifurcation model", J. Biomech., 38(6), pp. 1283-1290 (2005).

22. Prosi, M., Perktold, K., Ding, Z. and Friedman, M.H. "Influence of curvature dynamics on pulsatile coronary artery flow in a realistic bifurcation model", J. Biomech., 37(11), pp. 1767-1775 (2004).

23. Kabinejadian, F. and Ghista, D.N. "Compliant model of a coupled sequential coronary arterial bypass graft: effects of vessel wall elasticity and non-Newtonian rheology on blood flow regime and hemodynamic parameters distribution", Med. Eng. Phys., 34(7), pp. 860-872 (2012).

24. Dodge, J., Brown, B.G., Bolson, E.L. and Dodge, H.T. "Lumen diameter of normal human coronary arteries. Influence of age, sex, anatomic variation, and left ventricular hypertrophy or dilation", Circulation, 86(1), pp. 232-246 (1992).

25. Gradus-Pizlo, I., Bigelow, B., Mahomed, Y., Sawada, S.G., Rieger, K. and Feigenbaum, H. "Left anterior descending coronary artery wall thickness measured by high-frequency transthoracic and epicardial echocardiography includes adventitia", The American Journal of Cardiology, 91(1), pp. 27-32 (2003).

26. Ding, Z. and Friedman, M.H. "Quantification of 3D coronary arterial motion using clinical biplane cineangiograms", The International Journal of Cardiac Imaging, 16(5), pp. 331-346 (2000). 
27. Ramaswamy, S., Vigmostad, S., Wahle, A., Lai, Y.-G., Olszewski, M., Braddy, K., Brennan, T., Rossen, J., Sonka, M. and Chandran, K. "Fluid dynamic analysis in a human left anterior descending coronary artery with arterial motion", Ann. Biomed. Eng., 32(12), pp. 1628-1641 (2004).

28. Malvè, M., Garcia, A., Ohayon, J. and Martinez, M. "Unsteady blood flow and mass transfer of a human left coronary artery bifurcation: FSI vs. CFD", International Communications in Heat and Mass Transfer, 39(6), pp. 745-751 (2012).

29. Tang, D., Yang, C., Kobayashi, S., Zheng, J., Woodard, P.K., Teng, Z., Billiar, K., Bach, R. and $\mathrm{Ku}$, D.N. "3D MRI-based anisotropic FSI models with cyclic bending for human coronary atherosclerotic plaque mechanical analysis", J. Biomech. Eng., 131(6), pp. 061010 (2009).

30. Liu, Y., Lai, Y., Nagaraj, A., Kane, B., Hamilton, A., Greene, R., Mcpherson, D. and Chandran, K. "Pulsatile flow simulation in arterial vascular segments with intravascular ultrasound images", Med. Eng. Phys., 23(8), pp. 583-595 (2001).

31. Davies, J.E., Whinnett, Z.I., Francis, D.P., Manisty, C.H., Aguado-Sierra, J., Willson, K., Foale, R.A., Malik, I.S., Hughes, A.D. and Parker, K.H. "Evidence of a dominant backward-propagating "suction" wave responsible for diastolic coronary filling in humans, attenuated in left ventricular hypertrophy", Circulation, 113(14), pp. 1768-1778 (2006).

32. Huo, Y., Choy, J.S., Svendsen, M., Sinha, A.K. and Kassab, G.S. "Effects of vessel compliance on flow pattern in porcine epicardial right coronary arterial tree", J. Biomech., 42(5), pp. 594-602 (2009).

33. Crosetto, P., Reymond, P., Deparis, S., Kontaxakis, D., Stergiopulos, N. and Quarteroni, A. "Fluidstructure interaction simulation of aortic blood flow", Computers \& Fluids, 43(1), pp. 46-57 (2011).

34. Janela, J., Moura, A. and Sequeira, A. "A 3D nonNewtonian fluid-structure interaction model for blood flow in arteries", Journal of Computational and Applied Mathematics, 234(9), pp. 2783-2791 (2010).

35. Wang, X. and Li, X. "Biomechanical behaviors of curved artery with flexible wall: A numerical study using fluid-structure interaction method", Comput. Biol. Med., 41(11), pp. 1014-1021 (2011).

36. Valencia, A. and Baeza, F. "Numerical simulation of fluid-structure interaction in stenotic arteries considering two layer nonlinear anisotropic structural model", International Communications in Heat and Mass Transfer, 36(2), pp. 137-142 (2009).
37. Valencia, A. and Villanueva, M. "Unsteady flow and mass transfer in models of stenotic arteries considering fluid-structure interaction", International Communications in Heat and Mass Transfer, 33(8), pp. 966-975 (2006).

38. Qiu, Y. and Tarbell, J.M. "Numerical simulation of pulsatile flow in a compliant curved tube model of a coronary artery", J. Biomech. Eng., 122(1), pp. 77-85 (2000).

39. Sadeghi, M., Shirani, E., Tafazzoli-Shadpour, M. and Samaee, M. "The effects of stenosis severity on the hemodynamic parameters-assessment of the correlation between stress phase angle and wall shear stress", J. Biomech., 44(15), pp. 2614-2626 (2011).

40. Goubergrits, L., Wellnhofer, E., Kertzscher, U., Affeld, K., Petz, C. and Hege, H.-C. "Coronary artery WSS profiling using a geometry reconstruction based on biplane angiography", Ann. Biomed. Eng., 37(4), pp. 682-691 (2009).

41. Vavourakis, V., Papaharilaou, Y. and Ekaterinaris, J. "Coupled fluid-structure interaction hemodynamics in a zero-pressure state corrected arterial geometry", $J$. Biomech., 44(13), pp. 2453-2460 (2011).

\section{Biographies}

Hossein Ali Pakravan received his MSs degree in energy conversion, in 2010, from Shiraz University, Iran. and is currently a PhD candidate in Mechanical Engineering at Sharif University of Technology, Tehran, Iran. His research interests are primarily in the simulation of cardiovascular systems and cell mechanics.

Mohammad Said Saidi is Professor of Mechanical Engineering at Sharif University of Technology, Tehran, Iran. His research interests include modeling and numerical analysis of transport and deposition of aerosol particles, modeling and numerical analysis of biofluids, modeling and numerical analysis of thermalhydraulics of porous media and microchannels.

Bahar Firoozabadi is Professor of Mechanical Engineering at Sharif University of Technology, Tehran, Iran. Her research interests include modeling and numerical analysis of biofluids, particle-laden flow, and density current in settling tanks. 